\title{
Laser Enhanced Combinatorial Chemo-photothermal Therapy of Green Synthesis Gold Nanoparticles Loaded with 6Mercaptopurine on Breast Cancer Model
}

\author{
Amna H. Faid ${ }^{1}$. Samia A. Shouman ${ }^{2} \cdot$ Nadia A. Thabet ${ }^{2} \cdot$ Yehia A. Badr $^{1} \cdot$ Mahmoud A. Sliem $^{1,3}$
}

Accepted: 10 February 2022

(c) The Author(s) 2022

\begin{abstract}
Purpose We tend to evaluated a method for loading 6Mercaptopurine (6MP) on green synthesized hybrid chitosan gold nanoparticles (AuNPs) forming 6MP-AuNPs nanocomposite for the first time in combinatorial chemo-photothermal therapy. Methods The AuNPs were synthesized using chitosan as a reducing and capping agent. Different concentrations of 6MP were mixed AuNPs. Cells were incubated with 6MP and 6MP loaded AuNPs for $48 \mathrm{~h}$ and then exposed to laser.

Results AuNPs and 6MP-AuNPs nanocomposite have small sizes of $18 \pm 4$ and $25 \pm 5 \mathrm{~nm}$ and exhibit high stability with Zeta potential of $55.9 \pm 6.3$ and $57 \pm 4 \mathrm{mV}$. 6MP-AuNPs nanocomposite irradiated with Diode Pumped Solid State (DPSS) laser showed a maximum inhibition in cell viability reaching $63 \%$ at $1.25 \mu \mathrm{M}$.

Conclusions A hybrid chitosan gold nanoparticle is a powerful anti-cancer drug carrier as well as photothermal agent in combinatorial chemo-photothermal therapy.
\end{abstract}

Keywords Chitosan $\cdot$ Laser $\cdot$ Nanoparticles $\cdot$ Drug delivery $\cdot$ Nanocomposites $\cdot$ Photothermal chemotherapy

\section{Introduction}

Breast cancer (BC) is the central cause of cancer associated deaths of women universally. The main aim in the fight against cancer is developing effective therapeutic plans with low toxicities and high specificities to eliminate tumors

Amna H. Faid

amna_hussein090@yahoo.com

Samia A. Shouman

samia.shouman@nci.cu.edu.eg

Nadia A. Thabet

nadia_nci@yahoo.com

Yehia A. Badr

ybadr@niles.edu.eg

Mahmoud A. Sliem

mahmoud.ashour@niles.edu.eg

1 National Institute of Laser Enhanced Science, Cairo University, Giza, Egypt

2 National Cancer Institute (NCI), Cairo University, Cancer biology, Giza, Egypt

3 Chemistry Department, Faculty of Science and Arts, Al Ula Branch, Taibah University, Medina 41921, Saudi Arabia
$[1,2]$. Recently, photothermal therapy (PTT) has attracted significant attention as non-invasive cancer therapy [3] and selectively destroys cancer cells which are more sensitive to an increase in temperature [4]. Gold nanoparticles (AuNPs) attracted great attention due to their high localized surface plasmon resonance (LSPR) [5, 6]. Chitosan (CS) showed promising advantages in drug delivery systems, such as biocompatibility and biodegradability $[7,8]$. The $6 \mathrm{MP}$ is one of the most effective anti-cancer agents used to treat inflammatory diseases $[9,10]$. The use of $6 \mathrm{MP}$ is limited due to its poor bioavailability and short plasma half-life [11]. Combination therapy is considered to be a promising strategy for improving the therapeutic efficiency of cancer treatment [12]. A combination of AuNPs-based PTT and synchronized chemotherapy using a stimuli-responsive drug delivery system for controlled drug release has superior anticancer efficiency [13]. In the present study, we reported on the eco-friendly synthesis of AuNPs using chitosan as reducing and capping agent, and for the first time loading 6MP on chitosan reduced AuNPs forming 6MP-AuNPs nanocomposite. Moreover, the cytotoxic effects in addition to PTT and chemo-photothermal combined treatment on MCF7 were carried out. 


\section{Materials and Methods}

\section{Green Synthesis of Gold Nanoparticles by Chitosan (AuNPs)}

The AuNPs were synthesized using chitosan as a reducing and capping agent [14]. Varying chitosan concentrations $(0.05,0.01,0.1$, and 0.2$)$ were prepared in $1 \%$ acetic acid. A $10 \mu \mathrm{l}$ of $1.25 \times 10^{-1} \mathrm{M}$ solution of chloroauric acid (HAuCl4) was reduced by heating for $15 \mathrm{~min}$ in $10 \mathrm{ml}$ of chitosan.

\section{Preparation of 6MP Loaded AuNPs (6MP-AuNPs Nanocomposite)}

Different concentrations of 6MP $(1.25,2.5,5,10$, and $20 \mu \mathrm{M}$ ) were mixed drop wise with $1 \mathrm{ml}$ of the synthesized AuNPs and sonicated for $20 \mathrm{~min}$.

\section{Photothermal Chemotherapy Combined Treatment}

For laser irradiation, second harmonic Nd:YAG DPSS laser with wave length $532 \mathrm{~nm}$ and $200 \mathrm{~mW}$ was used. Cells were seeded according to that of Skehan et al. [15]. Cells were incubated with the same concentrations $1.25 \mu \mathrm{M}$ of free 6MP and 6MP loaded AuNPs for $48 \mathrm{~h}$ and then exposed to laser for different times: 2, 4, 6, and $8 \mathrm{~min}$. The experiment was repeated 3 times independently, and each concentration was repeated three times. Data are expressed as the arithmetic mean \pm SD. Statistical analysis was carried out using GraphPad Software Prism v5 (San Diego, USA). The statistical analysis of the transfection assays data was done using Tukey multiple comparison test One-way analysis of variance (ANOVA) with a single pooled variance. Differences were considered statistically significant when $p \leq 0.01$.

\section{Result and Discussion}

\section{Characterization of AuNPs and 6MP-AuNPs Nanocomposite}

AuNPs exhibits surface plasmon band SPB at $522 \mathrm{~nm}$ [14]. At lower concentrations of CS $0.01 \% \mathrm{w} / \mathrm{v}$ resulted in agglomerated particles Fig. 1a, however, concentrations of $0.05 \% \mathrm{w} / \mathrm{v}$ and above showed no agglomeration. This was reflected in SPB, which showed shift from $522 \mathrm{~nm}$ to $530 \mathrm{~nm}$ with $0.01 \% \mathrm{w} / \mathrm{v}$ which may be due to at lower concentration the particle charge is insufficient and lead to sensitization of the particles causing agglomeration, However, at higher concentrations $(>0.05 \% \mathrm{w} / \mathrm{v})$, the particles charge is sufficient for making them electrostatically stable. The absorption peaks of CS and AuNPs are shown in Fig. 1b. CS has a characteristic absorption peak at $300 \mathrm{~nm}$, and the absence of CS absorption peak clearly indicates that all its amino groups are actively involved in the reduction process [16]. The
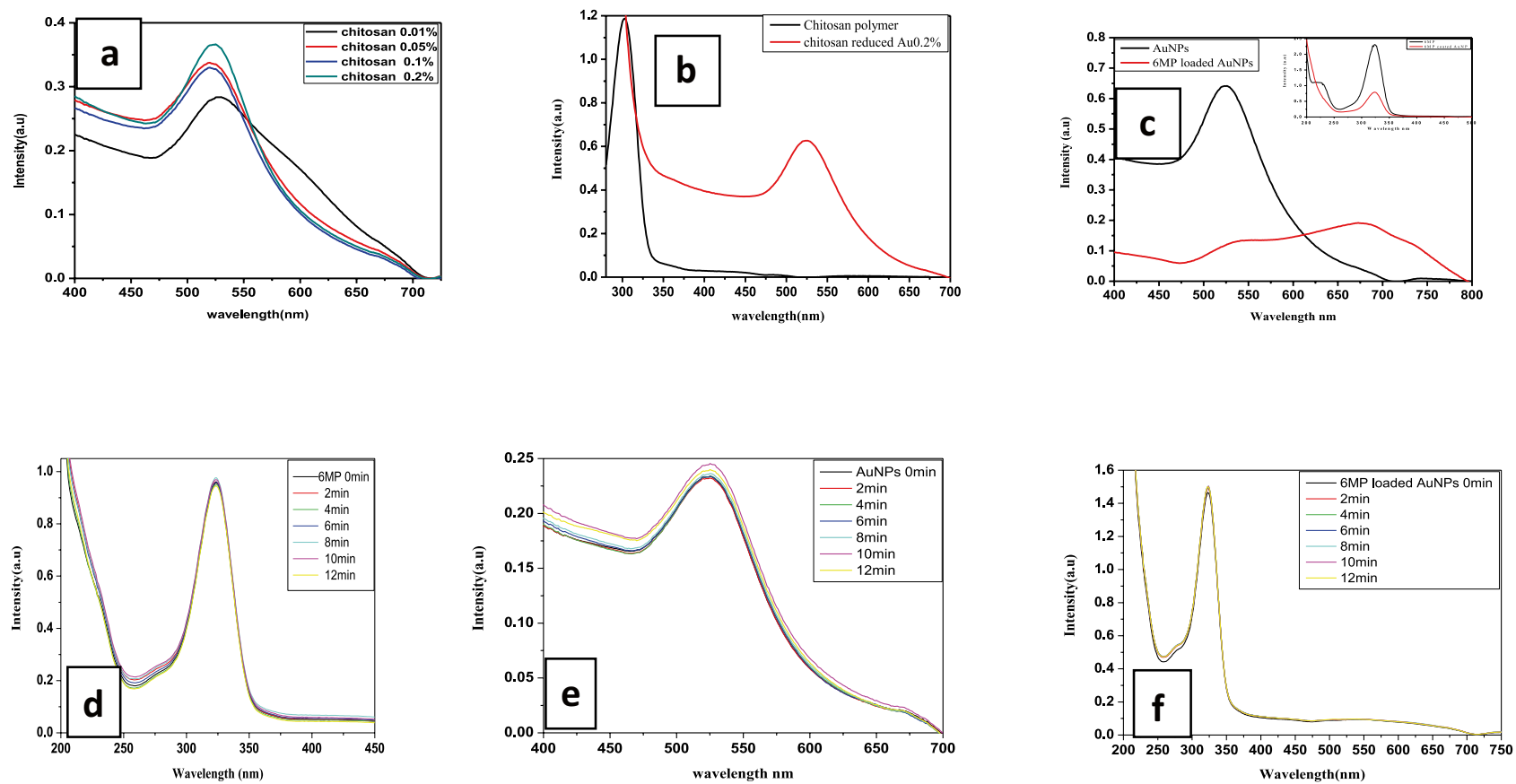

Fig. 1 UV-visible spectra of a AuNPs using various concentrations of CS, b CS polymer and $0.2 \%$ AuNPs, and $\mathbf{c} 0.2 \%$ AuNPs and $6 \mathrm{MP}-A u N P$. The inset view is the $\mathrm{n}-\pi^{*}$ transition of $6 \mathrm{MP}$ and $6 \mathrm{MP}-$
AuNPs and $\mathbf{d}$ free 6MP, e AuNPs, and 6MP f 6MP-AuNPs at different laser exposures 
interaction between 6MP and $0.2 \%$ chitosan reduced AuNPs was investigated via UV-visible spectroscopy, Fig. 1c, the inset view is a band in UV region appeared at $321 \mathrm{~nm}$ arises from the $n-\pi^{*}$ transition of $6 \mathrm{MP}$ molecule [17]. Upon addition of 6MP to AuNPs, the absorption spectrum showed a slight red shift from 522 to $528 \mathrm{~nm}$ with a decrease in absorbance intensity. At higher wavelength, another new band was observed around $680 \mathrm{~nm}$. This band is due to interparticle interaction between the adjacent AuNPs upon the addition of $6 \mathrm{MP}[18,19]$. Upon laser irradiation, the absorption spectra of AuNPs, 6MP, and 6MP loaded AuNPs showed no change in absorption bands which indicates the stability of the new nanocomposite toward laser at this time interval.

TEM images have uniform spherical shape and size range from 15 to $50 \mathrm{~nm}$ (Fig. 2a-d). At low CS, 0.05\% and $0.01 \% \mathrm{w} / \mathrm{v}$ aggregated particles were observed. TEM images of 6MP loaded on $0.2 \%$ chitosan reduced AuNPs Fig. 2e; reveals that nanocomposite possessed a regularly spherical shape and smooth surface with increases in the particle size from $18 \pm 4 \mathrm{~nm}$ to $25 \pm 5 \mathrm{~nm}$. Here, the plasmon coupling appeared clearly with linearly arranged coupled particles.

To determine the surface charges and stability of the prepared AuNPs, zeta potential was measured the higher the zeta potential, the more stable the nanoparticles due to the stronger repulsive force between each other [20,21]. AuNPs reduced by $0.2 \%$ CS showed the highest zeta potential with small size and uniform size distribution, so this concentration was chosen for 6MP loading. The shift in zeta potential from 55 to $57 \mathrm{mV}$ in case of loading 6MP on AuNPs indicates the high stability of the prepared nanocomposite.

Figure 2f, FTIRs featuring of CS and AuNPs. The main vibrational bands of CS are $3436 \mathrm{~cm}^{-1}$ is attributed to hydrogen-bonded $\mathrm{O}-\mathrm{H}$ stretching vibration; bands of $\mathrm{N}-\mathrm{H}$ stretching from primary amine and type II amide are overlapped in the same region [22], this band becomes sharper with shift to $3429 \mathrm{~cm}^{-1}$ in AuNPs. Band at $1652 \mathrm{~cm}^{-1}$ corresponding to amide I band characteristic to $\mathrm{C}=\mathrm{O}$ stretching of $\mathrm{N}$-acetyl group was found to be shifted
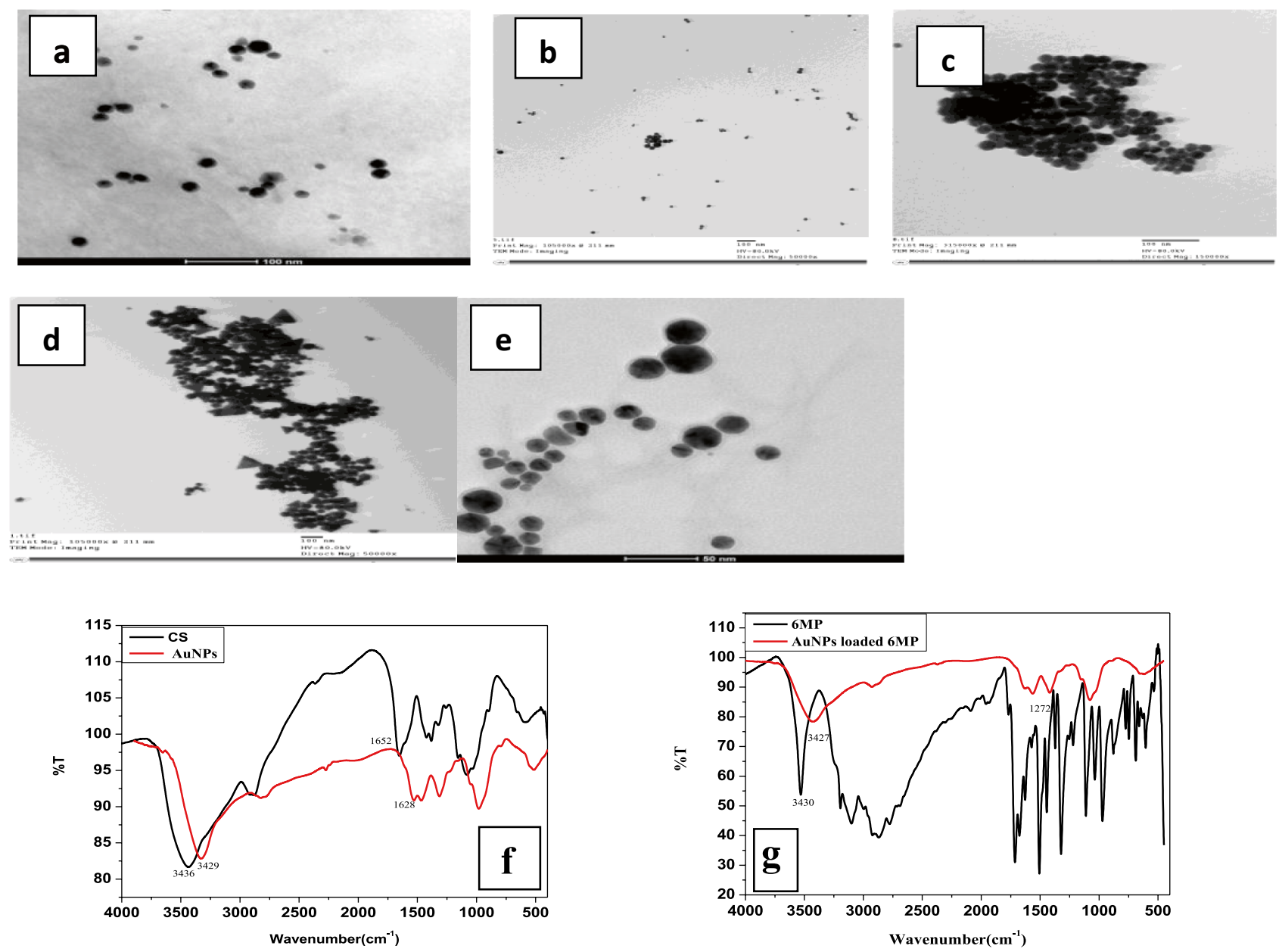

Fig. 2 TEM images of AuNPs using varying concentrations of CS a $0.2 \%, \mathbf{b} 0.1 \%, \mathbf{c} 0.05 \%$, and d $0.01 \%$ w/v, respectively; e TEM images of $6 \mathrm{MP}$ loaded on $0.2 \%$ w/v chitosan AuNPs. FTIR spectra of $\mathbf{f}$ chitosan and Au NPs and $\mathbf{g} 6 \mathrm{MP}$ and 6MP-AuNPs nanocomposite 


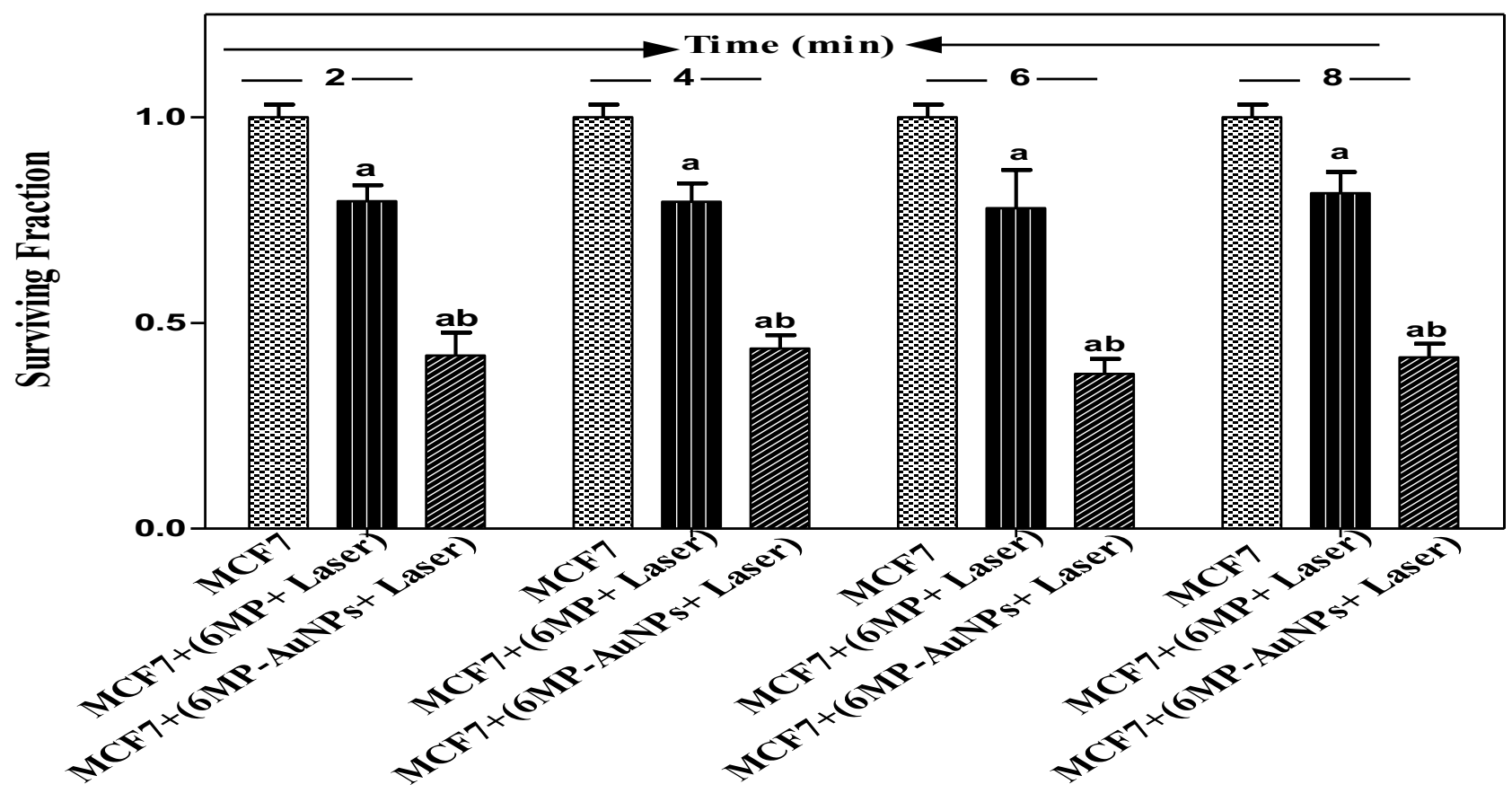

Fig. 3 The effect of DPSS laser irradiation MCF7 incubated with 6MP and 6MP loaded AuNPs

to $1628 \mathrm{~cm}^{-1}$ for AuNPs which clearly indicates that AuNPs are completely bounded with CS through affinity interaction with amino group [23]. The results prove the involvement of hydroxyl and primary amino groups of CS in AuNPs reduction and stabilization. Figure $2 \mathrm{~g}$, FTIR spectrum of 6MP shows characteristic bands at 3430.74, 1527.35, 3094.23, 2999.73, 1344.14, 1613.16, 1668.12, 2676.71, $1154.19,1120.44,1120.44$ and $1272.79 \mathrm{~cm}^{-1}$ corresponding to combined peaks of the $\mathrm{NH} 2$ and $\mathrm{OH}$ group stretching vibration, $\mathrm{N}-\mathrm{H}$ (Bend), $\mathrm{C}-\mathrm{H}$ (stretch), $\mathrm{C}-\mathrm{N}, \mathrm{C}=\mathrm{N}, \mathrm{C}=\mathrm{C}$, $\nu \mathrm{C}=\mathrm{S} /$ ring vibration, $\nu \mathrm{C}=\mathrm{S} /$ ring vibration and $\mathrm{C}=\mathrm{S}$ stretching respectively [24]. In the case of loading 6MPAuNPs there were a decrease in intensity of all bands, band at $3430 \mathrm{~cm}^{-1}$ corresponding to combined $\mathrm{NH} 2$ and $\mathrm{OH}$ group stretching vibration is broadened and slightly shifted to lower wavelengths $3427 \mathrm{~cm}^{-1}$ confirms formation of complex 6MP-AuNPs nanocomposite. In addition, the disappearance of $6 \mathrm{MP}$ characteristic peak at $1272 \mathrm{~cm}^{-1}$ corresponding to $\mathrm{C}=\mathrm{S}$ stretching confirming the existence of complex formation of the 6MP with AuNPs through the sulphur atom [25].

\section{Photothermal Chemotherapy Combined Treatment}

Cell viability after laser irradiation showed a decrease in cell survival by $24 \%, 21 \%, 33 \%$, and $19 \%$ for free $6 \mathrm{MP}$ and $58 \%$, $57 \%, 63 \%$, and $59 \%$ for $6 \mathrm{MP}-\mathrm{AuNPs}$ at $2,4,6$, and $8 \mathrm{~min}$, respectively, compared to $5 \%$ and $55 \%$ inhibition produced by $1.25 \mu \mathrm{M}$ of $6 \mathrm{MP}$ and $6 \mathrm{MP}-\mathrm{AuNPs}$, respectively, in the absence of laser irradiation (Fig. 3); the enhancement of 6MP cytotoxicity after laser irradiated may be due to that DNA repair processes which are the essential response for the cells to aggression by cytotoxic drugs are temperaturedependent [26]. In addition to the highlighting loss of cell membranes integrity, which are severely affected by heat shock, enhancing permeability leading to accumulation of higher concentration of the drug at tumor site [27]. The extensive cell death was observed for cells irradiated in presence of 6MP-AuNPs when compared to irradiation of free 6MP which indicates that the AuNPs are required for photothermal conversion of laser irradiation. By using localized hyperthermia combined with chemotherapy, a reduction of the dose with minimum side effect of 6MP can be used to achieve better therapeutic effect.

\section{Conclusion}

The present work demonstrated a method for fast, ecofriendly, highly stable, and low-cost synthesis of AuNPs using a biocompatible polymer chitosan. Combination of therapeutic approaches against cancer has become an essential step to improve outcome and prevent the appearance of resistance to chemotherapy. Future work is in progress to investigate the positive effects of chemo-photothermal combination using the prepared nanocomposite for in vivo cancer treatment. 
Acknowledgements We would like to acknowledge Dr. Elham Mostafa lecturer assistant at NILES Cairo university for helping this work.

Funding Open access funding provided by The Science, Technology \& Innovation Funding Authority (STDF) in cooperation with The Egyptian Knowledge Bank (EKB).

Open Access This article is licensed under a Creative Commons Attribution 4.0 International License, which permits use, sharing, adaptation, distribution and reproduction in any medium or format, as long as you give appropriate credit to the original author(s) and the source, provide a link to the Creative Commons licence, and indicate if changes were made. The images or other third party material in this article are included in the article's Creative Commons licence, unless indicated otherwise in a credit line to the material. If material is not included in the article's Creative Commons licence and your intended use is not permitted by statutory regulation or exceeds the permitted use, you will need to obtain permission directly from the copyright holder. To view a copy of this licence, visit http://creativecommons.org/licenses/by/4.0/.

\section{References}

1. Movahedi M, Haghighat S, Khayamzadeh M, Moradi A, GhanbariMotlagh A. Mirzaei H et al Survival rate of breast cancer based on geographical variation in Iran, a national study. Iran Red Crescent Med J. 2012;14(12):798.

2. Sheng W-Y, Huang L. Cancer immunotherapy and nanomedicine. Pharm Res. 2011;28(2):200-14.

3. Yang K, Yang G, Chen L, Cheng L, Wang L, Ge C. FeS nanoplates as a multifunctional nano-theranostic for magnetic resonance imaging guided photothermal therapy. Biomaterials. 2015;38:1-9.

4. Huang X, El-Sayed MA. Plasmonic photo-thermal therapy (PPTT), Alexandria. J Med. 2011;47:1-9.

5. Bai X, Wang Y, Song Z, Feng Y, Chen Y, Zhang D, Feng L. The basic properties of gold nanoparticles and their applications in tumor diagnosis and treatment Int. J Mol Sci. 2020;21:2480. https://doi.org/10.3390/ijms21072480.

6. Sharma H, Mishra PK, Talegaonkar S, Vaidya B. Metal nanoparticles: a theranosticnanotool against cancer. Drug Discov Today. 2015;20:1143-51.

7. Ma K, Cheng Y, Wei X, Chen D, Zhao X, Jia P. Gold embedded chitosan nanoparticles with cell membrane mimetic polymer coating for $\mathrm{pH}$-sensitive controlled drug release and cellular fluorescence imaging. J Biomater Appl. 2021;35(7):857-68.

8. Chen J, Dong X, Feng T. Charge-conversional zwitterionic copolymer as $\mathrm{pH}$-sensitive shielding system for effective tumor treatment. Acta Biomater. 2015;26:45-53.

9. Cuin A, Massabni AC, Pereira GA, Leite CQF, Pavan FR, Costa RS, Heinrich TA, Costa-Neto CM. 6-mercaptopurine complexes with silver and gold ions: anti-tuberculosis and anti-cancer activities. Biomed Pharmacother. 2011;65:334-8.

10. Sun H, Wang T, Liu X, Chen P. A sensitive inhibition chemiluminescence method for the determination of 6-mercaptopurine in tablet and biological fluid using the reaction of luminol-Ag (III) complex in alkaline medium. J Lumin. 2013;134:154-9.

11. Kevadiya BD, Patel TA, Jhala DD, Thumbar RP, Brahmbhatt H, Pandya MP, Rajkumar S, Jena PK, Joshi GV, Gadhia PK, Tripathi CB. Layered inorganic nanocomposites: a promising carrier for 5-fluorouracil (5-FU). Eur J Pharma Biopharm. 2012;81(1):91-101.
12. Yoo E, Choi JH, Hoang NH, Lee JS, Vuong S, Hur B, Han P, Oh KT, Fahmy T, Kim D. Particle-in-particle platform for nanoconfinement-induced oncothermia. ACS Appl Bio Mater. 2018;1:1927-41.

13. Sim T, Lim C, Hoang NH, Shin Y, Kim JC, Park JY, Her J, Lee $\mathrm{ES}$, Youn YS, Oh KT. An on-demand $\mathrm{pH}$-sensitive nanocluster for cancer treatment by combining photothermal therapy and chemotherapy. Pharmaceutics. 2020;12(9):839.

14. Malathi S, Balakumaran MD, Kalaichelvan PT, Balasubramanian S. Green synthesis of gold nanoparticles for controlled delivery. Adv Mat Lett. 2013;4(12):933-40.

15. Skehan P, Storeng R, Scudiero D, Monks A, McMahon VD, Warren JT, Bokesch H, Kenney S, Boyd MR. New colorimetric cytotoxicity assay for anticancer-drug screening. J Natl Cancer Inst. 1990;82:1107-12.

16. Roshdi S, Doaa AS. Studies on the effect of the capping materials on the spherical gold nanoparticles catalytic activity. World J Nano Sci Eng. 2011;1:51-61.

17. Narayanan RL, Sivakumar M. Preparation and characterization of gold nanoparticles in chitosan suspension by one-pot chemical reduction method. Nano Hybrids. 2014;6:47-57.

18. Mohammad HS, Mohammad YK, Kaveh A, Abdolhossein N, Davoud A, Mohammad RR. Simultaneous determination of 6-Mercaptopurine and its oxidative metabolites in synthetic solutions and human plasma using spectrophotometric multivariate calibration methods. BioImpacts. 2011;1(1):53-62.

19. Tom RT, Suryanarayanan V, Reddy GP, Baskaran S, Pradeep T. Ciprofloxacin protected gold nanoparticles. Langmuir. 2004;20:1909-14.

20. Zhanguang C, Zhen W, Xi C, Haixiong X, Jinbin L. Chitosan-capped gold nanoparticles for selective and colorimetric sensing of heparin. JNanopart Res. 2013;15:1930-5.

21. Acevedo CR, Costas ME, Escudero R. Magnetic study of the novel polynuclear compound [Cu (II)(6-mercaptopurinolate2-) ]n. J Solid State Chem. 1997;132(1):78-87.

22. Love JC, Estroff LA, Kriebel JK, Nuzzo RG, Whitesides GM. Self-assembled monolayers of thiolates on metals as a form of nanotechnology. Chem Rev. 2005;105:1103-69.

23. Kaur G, Behrsing H, Parchment RE, Millin MD, Teicher BA. Analyses of the combination of 6-MP and dasatinib in cell culture. Int J Oncol. 2013;43(1):13-22.

24. Munshi PN, Lubin M, Bertino JR. 6-thioguanine: a drug with unrealized potential for cancer therapy. The Oncologist. 2014;19(7):760-5

25. Sadaf A, Reza Y, Reza F, Abdolkarim A, Ali N, MansoorehAhmad $\mathrm{JH}$. The improvement of anti-proliferation activity against breast cancer cell line of thioguanine by gold Nanoparticles. Med Chem Res. 2012. https://doi.org/10.1007/s00044-012-0030-12.

26. Agabeigi R, Rasta SH, Rahmati-Yamchi M, Salehi R, Alizadeh E. 1, Novel chemo-photothermal therapy in breast cancer using methotrexate-loaded folic acid conjugated $\mathrm{Au} @ \mathrm{SiO} 2$ nanoparticles. Nanoscale Res Lett. 2020;15:62.

27. Fernandes AR. Multifunctional gold-nanoparticles: a nanovectorization tool for the targeted delivery of novel chemotherapeutic agents. J Control Release. 2017;245:52-61.

Publisher's Note Springer Nature remains neutral with regard to jurisdictional claims in published maps and institutional affiliations. 